\title{
Periodic Solutions of Autonomous Functional-Differential Equations with State Dependent Deviations
}

\author{
By \\ Svatoslav STANĚK* \\ (Palacký University, Czech Republic)
}

\begin{abstract}
In this paper, we consider periodic solutions of the functional-differential equation $x^{\prime \prime}+x(t-k x)=0$. The structure of the set $\mathscr{A}_{k}(k \in(0, \infty))$ of all its nontrivial periodic solutions $x$ satisfying $x^{\prime}<1 / k$ on $\boldsymbol{R}$ is described. It is proved that for each $k \in(0, \infty)$ and $T_{*} \in(2 \pi, \infty)$, there exists $x \in \mathscr{A}_{k}$ having the period $T_{*}$ and for each $k \in(0, \infty)$ and $a \in(0,1 / k)$, there exists a unique $x \in \mathscr{A}_{k}$ such that $x(0)=0$ and $x^{\prime}(0)=a$.

Key Words and Phrases. Second order functional-differential equation, State dependent deviation, Periodic solution.

2000 Mathematics Subject Classification Numbers. 34K13.
\end{abstract}

\section{Introduction}

In this paper, we discuss periodic solutions $x$ of the functional-differential equation

$$
x^{\prime \prime}(t)+x(t-k x(t))=0, \quad k \in(0, \infty)
$$

for which

$$
x^{\prime}(t)<\frac{1}{k}, \quad t \in \boldsymbol{R}
$$

We say that a function $x \in C^{2}(\boldsymbol{R})$ is a periodic solution of problem (1.1), (1.2) if $x$ satisfies (1.1) and (1.2) for $t \in \boldsymbol{R}, x \neq 0$ and $x$ is a periodic function.

If $x$ is a periodic solution of problem (1.1), (1.2) with a period $P$, then $x$ changes its sign on any interval of the length $P$. (By the period $P$ of a nonconstant periodic function $x$ we mean the number $P=\inf \{a: a \in(0, \infty)$, $x(t+a)=x(t)$ for $t \in \boldsymbol{R}\}$.) Otherwise $x \geq 0$ or $x \leq 0$ on $\boldsymbol{R}$ and then $x^{\prime \prime} \leq 0$ or $x^{\prime \prime} \geq 0$ on $\boldsymbol{R}$ which implies that $x^{\prime}$ is a monotone function and since $x$ is $P$ periodic, $x^{\prime}=0$. Hence $x$ is a constant function and from $x^{\prime \prime}(t)+x(t-k x(t))$ $=0$ we see that $x=0$, which is a contradiction.

\footnotetext{
* Supported by grant no. 201/04/1077 of the Grant Agency of Czech Republic and by the Council of Czech Government J14/98:153100011
} 
Notice that (1.1) is a functional-differential equation with a deviation depending on solutions. Since any periodic solution of (1.1) changes its sign, equation (1.1) does not belong to the class of functional-differential equations with state dependent delay. In the literature periodic solutions are considered for functional-differential equations with state dependent delays of the form $r(t, x)$ where $r$ is nonnegative and bounded on a subset of $\boldsymbol{R}^{2}$. For the first-order scalar functional-differential equations see e.g. [1], [2], [4] and references therein. But in [5] and [6] periodic solutions are considered for iterative functional-differential equations, which belong to the class of functional-differential equations with a state dependent deviation. In [5] the existence and properties of the first-order iterative functional-differential equation $x^{\prime}=\omega(t)(a x-b x(x)), a>b>0$, are studied. Conditions for the existence of periodic solutions of the second-order functional-differential equation $x^{\prime \prime}+g(x(x))=p(t)$ are given in [6]. Remember also paper [3] where lower bounds for periods of periodic solutions to an autonomous iterative functional-differential equation

$$
x^{\prime}(t)=G\left(x(t), x^{[2]}(t), \ldots, x^{[m]}(t)\right)
$$

are presented. Here $G: \boldsymbol{R}^{m} \rightarrow \boldsymbol{R}$ satisfies a Lipschitz condition and $x^{[i]}(t)=$ $(x \circ \cdots \circ x)(t)$ is the $i$ th iteration of $x$.

For $k \in(0, \infty)$, denote by $\mathscr{A}_{k}$ the set of all periodic solutions $x$ of problem (1.1), (1.2).

The aim of the paper is to consider properties and the structure of the set $\mathscr{A}_{k}, k \in(0, \infty)$.

Together with problem (1.1), (1.2), we consider the associated problem

$$
\begin{gathered}
y^{\prime \prime}(t)=y(t)-y(y(t)), \\
y^{\prime}(t)>0, \quad t \in \boldsymbol{R},
\end{gathered}
$$

and denote by $\Omega$ the set of all solutions $y$ on $\boldsymbol{R}, y(t) \not \equiv t$, to problem (1.3), (1.4) for which the function $x(t)=y(t)-t$ is periodic.

The discussion of periodic solutions to problem (1.1), (1.2), that is the discussion of the structure of the set $\mathscr{A}_{k}$, is based on the following result.

Lemma 1.1. Let $k \in(0, \infty)$. Then $x \in \mathscr{A}_{k}$ if and only if the function

$$
y(t)=t-k x(t), \quad t \in \boldsymbol{R},
$$

belongs to $\Omega$.

Proof. Let $x \in \mathscr{A}_{k}$ and $y$ be defined by (1.5). Then $y^{\prime}>0$ on $\boldsymbol{R}, t-y(t)$ is periodic, $t-y(t) \not \equiv 0$ and

$$
y^{\prime \prime}(t)=-k x^{\prime \prime}(t)=k x(t-k x(t))=k x(y(t))=y(t)-y(y(t)), \quad t \in \boldsymbol{R},
$$

which shows that $y$ is a solution of (1.3). Hence $y \in \Omega$. 
Let $y \in \Omega$ and set $x(t)=(t-y(t)) / k, t \in \boldsymbol{R}$. Then $x$ is periodic, $x^{\prime}=$ $\left(1-y^{\prime}\right) / k<1 / k$ and

$$
\begin{aligned}
x^{\prime \prime}(t) & =-\frac{y^{\prime \prime}(t)}{k}=-\frac{1}{k}[y(t)-y(y(t))] \\
& =-\frac{y(t)}{k}+\left(\frac{y(t)}{k}-x(y(t))\right)=-x(t-k x(t))
\end{aligned}
$$

for $t \in \boldsymbol{R}$. Therefore $x \in \mathscr{A}_{k}$.

The rest of the paper is organized as follows. Section 2 is devoted to the study of properties of solutions of (1.3). In Section 3, we introduce a function $T(\alpha),(\alpha \in(0,1))$ by a (unique) solution $y$ of $(1.3)$ satisfying the initial conditions $y(0)=0, y^{\prime}(0)=\alpha$, and consider properties of the function $T(\alpha)$ and the set $\Omega$. Main results of the paper are given in Section 4. It is described the structure of the set $\mathscr{A}_{k}$ and it is proved that for any $k>0$ and each $T_{*} \in(2 \pi, \infty)$, there exists a $T_{*}$-periodic solution of problem (1.1), (1.2). Also it is shown that for any $k>0$ and $a \in(0,1 / k)$, there exists a unique periodic solution $x$ of problem (1.1), (1.2) satisfying $x(0)=0, x^{\prime}(0)=a$.

\section{Properties of solutions of $y^{\prime \prime}(t)=y(t)-y(y(t))$}

The next lemma is obvious.

Lemma 2.1. Let $y(t)$ be a solution of (1.3) on an interval $J$. Then

$$
y: J \rightarrow J .
$$

Let $y(t)$ and $z(t)$ be solutions of (1.3) on intervals $J$ and $I$, respectively. We say that $z(t)$ is a continuation of $y(t)$ if $J \subset I, J \neq I$, and $y(t)=z(t)$ for $t \in J$. If $t \geq s(t \leq s)$ for $t \in I \backslash J$ and any $s \in J$, then we say that $z(t)$ is a right (left) continuation of $y(t)$. If a solution $y(t)$ of (1.3) has no continuation, then $y(t)$ is said to be a maximal solution of (1.3).

It is easily seen that for each solution $y$ of (1.3) there exists a maximal solution of (1.3) which is a continuation of $y$.

Lemma 2.2. Let $y(t)$ be a maximal solution of (1.3) on an interval $J$. Then for each $c \in \boldsymbol{R}$ and $\mu \in\{-1,1\}$, the function

$$
z_{\mu, c}(t)=c+\mu y(\mu(t-c)), \quad t \in I_{\mu, c}=\{t: \mu(t-c) \in J\},
$$

is a maximal solution of (1.3) on the interval $I_{\mu, c}$.

Proof. Let $c \in \boldsymbol{R}, \mu \in\{-1,1\}$ and $z_{\mu, c}$ be defined by (2.1). Then 


$$
\begin{aligned}
z_{\mu, c}^{\prime \prime}(t) & =\mu y^{\prime \prime}(\mu(t-c))=\mu[y(\mu(t-c))-y(y(\mu(t-c)))] \\
& =z_{\mu, c}(t)-c-\mu y\left(\mu\left(z_{\mu, c}(t)-c\right)\right) \\
& =z_{\mu, c}(t)-z_{\mu, c}\left(z_{\mu, c}(t)\right)
\end{aligned}
$$

for $t \in I_{\mu, c}$. Hence $z_{\mu, c}$ is a solution of (1.3) on $I_{\mu, c}$.

If $z_{\mu, c}$ is not a maximal solution of (1.3) for some $c \in \boldsymbol{R}$ and $\mu \in\{-1,1\}$, then there is a continuation $z$ of $z_{\mu, c}$ on an interval $I, I_{\mu, c} \subset I, I_{\mu, c} \neq I$. Therefore the function $u(t)=\mu[z(c+\mu t)-c], t \in J_{*}=\{t: c+\mu t \in I\}$, is a continuation of $y$, which is impossible.

Lemma 2.3. Let $y(t)$ be a maximal solution of (1.3) on an interval $J$. Then there exists $t_{0} \in J$ such that

$$
y\left(t_{0}\right)=t_{0} .
$$

Proof. First assume $y(t)<t$ for $t \in J$. Then Lemma 2.1 implies that $y(y(t))<y(t)$ for $t \in J$ which gives $y^{\prime \prime}>0$ on $J$ and $y^{\prime}$ is increasing. From $y(t)-y(y(t))=y^{\prime}(\xi)(t-y(t))$ for $t \in J$ with a $\xi \in(y(t), t)$, we see that $y^{\prime}(\xi)>0$. Since $y^{\prime}$ is increasing and $\xi<t$, we have $0<y^{\prime}(\xi)<y^{\prime}(t)$. Hence $y^{\prime}>0$ on $J$. We now show that $J=\boldsymbol{R}$. Let

$$
b=\sup \{t: t \in J\}<\infty .
$$

If $b \in J$ then $y(b)<b$, and consider the initial value problem

$$
\left\{\begin{array}{l}
z^{\prime \prime}=z-y(z) \\
z^{(j)}(b)=y^{(j)}(b), \quad j=0,1 .
\end{array}\right.
$$

Since $y \in C^{2}(J)$, there exists a unique solution $z$ of (2.3) on a neighbourhood $\mathscr{U}$ of $t=b$. Clearly, $z(t)=y(t)$ for $t \in J \cap \mathscr{U}$ and the function $w: J \cup \mathscr{U} \rightarrow \boldsymbol{R}$ defined by

$$
w(t)= \begin{cases}y(t) & \text { for } t \in J \\ z(t) & \text { for } t \in \mathscr{U} \backslash J\end{cases}
$$

is a solution of (1.3) on $J \cup \mathscr{U}$. Therefore $w$ is a right continuation of $y$, which is impossible due to the fact that $y$ is a maximal solution of (1.3). Let $b \notin J$. Since $y$ and $y^{\prime}$ are increasing on $J$, there exist $\lim _{t \rightarrow b^{-}} y(t)=A$ and $\lim _{t \rightarrow b^{-}} y^{\prime}(t)=B$. By Lemma 2.1, $A \leq b$. Since

$$
\lim _{t \rightarrow b^{-}} y^{\prime \prime}(t)=A-\lim _{t \rightarrow b^{-}} y(y(t))=A-\lim _{t \rightarrow A^{-}} y(t)
$$

and

$$
\lim _{t \rightarrow A^{-}} y(t)= \begin{cases}A & \text { if } A=b \\ y(A) & \text { if } A<b\end{cases}
$$


we see that $\lim _{t \rightarrow b^{-}} y^{\prime \prime}(t)$ is a finite number and $B \in(0, \infty)$. Set

$$
u(t)= \begin{cases}y(t) & \text { for } t \in J \\ A & \text { for } t=b\end{cases}
$$

Then

$$
\lim _{t \rightarrow b^{-}}(y(t)-y(y(t)))= \begin{cases}0 & \text { if } A=b \\ A-y(A) & \text { if } A<b\end{cases}
$$

and

$$
u(b)-u(u(b))=A-u(A)= \begin{cases}0 & \text { if } A=b \\ A-y(A) & \text { if } A<b .\end{cases}
$$

Letting $t \rightarrow b^{-}$in the equality $\left(t_{0} \in J\right)$

$$
y(t)=y\left(t_{0}\right)+y^{\prime}\left(t_{0}\right)\left(t-t_{0}\right)+\int_{t_{0}}^{t}(t-s)[y(s)-y(y(s))] d s, \quad t \in J,
$$

we get

$$
u(b)=y\left(t_{0}\right)+y^{\prime}\left(t_{0}\right)\left(b-t_{0}\right)+\int_{t_{0}}^{b}(b-s)[u(s)-u(u(s))] d s
$$

Hence

$$
u(t)=y\left(t_{0}\right)+y^{\prime}\left(t_{0}\right)\left(t-t_{0}\right)+\int_{t_{0}}^{t}(t-s)[u(s)-u(u(s))] d s, \quad t \in J \cup\{b\},
$$

and $u$ is a solution of (1.3) on $J \cup\{b\}$. Therefore $u$ is a right continuation of $y$, which is impossible. Consequently $b=\infty$. Similar reasoning as in the first part of the proof shows that $\inf \{t: t \in J\}=-\infty$. Hence $J=\boldsymbol{R}$. Therefore $y^{\prime}$ is increasing on $\boldsymbol{R}$. If $\lim _{t \rightarrow \infty} y^{\prime}(t)>1$, then there exist $t_{1}>0$ and $\gamma>1$ such that $y^{\prime}(t) \geq \gamma$ for $t \in\left[t_{1}, \infty\right)$. It follows that

$$
y(t)=y\left(t_{1}\right)+\int_{t_{1}}^{t} y^{\prime}(s) d s \geq y\left(t_{1}\right)+\gamma\left(t-t_{1}\right)
$$

and

$$
\frac{y(t)}{t} \geq \frac{y\left(t_{1}\right)}{t}+\gamma\left(1-\frac{t_{1}}{t}\right)
$$

for $t \in\left[t_{1}, \infty\right)$, which yields

$$
\liminf _{t \rightarrow \infty} \frac{y(t)}{t} \geq \gamma
$$


On the other hand from our assumption $y(t)<t$ for $t \in \boldsymbol{R}$ we get $y(t) / t<1$ for $t>0$, contrary to (2.4). Hence $\lim _{t \rightarrow \infty} y^{\prime}(t) \leq 1$ and then $y^{\prime}$ being increasing on $\boldsymbol{R}$ implies $y^{\prime}(t) \leq d$ for $t \in(-\infty,-1]$ with a $d<1$. Consequently,

$$
y(-1)-y(t)=\int_{t}^{-1} y^{\prime}(s) d s \leq-d(t+1)
$$

and

$$
\frac{y(t)}{t} \leq \frac{y(-1)}{t}+d\left(1+\frac{1}{t}\right)
$$

for $t \in(-\infty,-1]$. Hence

$$
\limsup _{t \rightarrow-\infty} \frac{y(t)}{t} \leq d
$$

But $y(t)<t$ for $t \in \boldsymbol{R}$ gives $\liminf _{t \rightarrow-\infty}(y(t) / t) \geq 1$, contrary to (2.5). Summarizing, we have shown that $y$ does not satisfy $y(t)<t$ for $t \in J$.

Assume now that $y(t)>t$ for $t \in J$. Set $v(t)=-y(-t)$ for $t \in I=$ $\{t:-t \in J\}$. Then $v$ is a maximal solution of (1.3) by Lemma 2.2 (see (2.1) with $c=0$ and $\mu=-1$ ). Since $y(-t)>-t$ for $t \in I$, we have $v(t)<t$ for $t \in I$. By the first part of the proof, this situation for $v$ is impossible, and so the inequality $y(t)>t$ for $t \in J$ is not true. We have proved that (2.2) holds with a $t_{0} \in J$.

Lemma 2.4. A function $y(t)$ is a solution of (1.3) on an interval $I$ and $y(0)=0, y^{\prime}(0)=\alpha$ if and only if $y(t)$ is a solution of the equation

$$
y(t)=\alpha \sinh t-\int_{0}^{t} \sinh (t-s) y(y(s)) d s
$$

in the set $C^{0}(I)$.

Proof. The validity of Lemma 2.4 follows immediately by a simple calculation.

Lemma 2.5. Let $\alpha \in(0,1)$. Then there exists a unique solution $y(t)$ of $(1.3)$ on an interval $[-\varepsilon, \varepsilon], \varepsilon>0$, such that

$$
y(0)=0, \quad y^{\prime}(0)=\alpha .
$$

Proof. Let $\varepsilon$ be a positive number satisfying the inequalities

$$
\begin{gathered}
\alpha \cosh \varepsilon+\varepsilon^{2} \cosh \varepsilon+\varepsilon \sinh (2 \varepsilon) \leq 1, \\
\varepsilon \sinh \varepsilon<\frac{1}{2}
\end{gathered}
$$


and set

$$
\mathscr{B}=\left\{x: x \in C^{0}([-\varepsilon, \varepsilon]), x(0)=0,\left|x\left(t_{2}\right)-x\left(t_{1}\right)\right| \leq\left|t_{2}-t_{1}\right| \text { for } t_{1}, t_{2} \in[-\varepsilon, \varepsilon]\right\} .
$$

Then $\mathscr{B}$ is a nonempty and closed subset of the Banach space $C^{0}([-\varepsilon, \varepsilon])$ endowed with the sup-norm $\|x\|=\max \{|x(t)|: t \in[-\varepsilon, \varepsilon]\}$.

For each $x \in \mathscr{B}$, we have

$$
|x(t)| \leq|t|, \quad t \in[-\varepsilon, \varepsilon],
$$

and therefore $x:[-\varepsilon, \varepsilon] \rightarrow[-\varepsilon, \varepsilon]$. Next, for $x, y \in \mathscr{B}$ and $t \in[-\varepsilon, \varepsilon]$,

$$
\begin{aligned}
|x(x(t))-y(y(t))| & \leq|x(x(t))-x(y(t))|+|x(y(t))-y(y(t))| \\
& \leq|x(t)-y(t)|+\|x-y\| \\
& \leq 2\|x-y\| .
\end{aligned}
$$

Define the operator $\mathscr{F}: \mathscr{B} \rightarrow C^{0}([-\varepsilon, \varepsilon])$ by

$$
(\mathscr{F} x)(t)=\alpha \sinh t-\int_{0}^{t} \sinh (t-s) x(x(s)) d s .
$$

By Lemma 2.4 and the Banach fixed point theorem, in order to prove our lemma it suffices to verify that

(i) $\mathscr{F}: \mathscr{B} \rightarrow \mathscr{B}$ and

(ii) $\mathscr{F}$ is contractive.

In order to prove (i), let $x \in \mathscr{B}$ and $t_{1}, t_{2} \in[-\varepsilon, \varepsilon]$. Then

$$
\begin{aligned}
\left|(\mathscr{F} x)\left(t_{2}\right)-(\mathscr{F} x)\left(t_{1}\right)\right| \\
=\mid \alpha\left(\sinh t_{2}-\sinh t_{1}\right)-\int_{0}^{t_{1}}\left[\sinh \left(t_{2}-s\right)-\sinh \left(t_{1}-s\right)\right] x(x(s)) d s \\
\quad-\int_{t_{1}}^{t_{2}} \sinh \left(t_{2}-s\right) x(x(s)) d s \mid \\
\leq \alpha\left|t_{2}-t_{1}\right| \cosh \xi+\left|\left(t_{2}-t_{1}\right) \int_{0}^{t_{1}} \cosh \eta x(x(s)) d s\right| \\
\quad+\left|\int_{t_{1}}^{t_{2}} \sinh \left(t_{2}-s\right) x(x(s)) d s\right|
\end{aligned}
$$

where $\xi$ lies between $t_{1}$ and $t_{2}$ and $\eta$ lies between $t_{2}-s$ and $t_{1}-s$. Now, by (2.6) and (2.8),

$$
\left|(\mathscr{F} x)\left(t_{2}\right)-(\mathscr{F} x)\left(t_{1}\right)\right| \leq\left[\alpha \cosh \varepsilon+\varepsilon^{2} \cosh \varepsilon+\varepsilon \sinh (2 \varepsilon)\right]\left|t_{2}-t_{1}\right| \leq\left|t_{2}-t_{1}\right|,
$$

which shows that (i) is true. 
Finally, for $x, y \in \mathscr{B}$, we have (see (2.9))

$$
|(\mathscr{F} x)(t)-(\mathscr{F} y)(t)|=\left|\int_{0}^{t} \sinh (t-s)[x(x(s))-y(y(s))] d s\right| \leq 2 \varepsilon \sinh \varepsilon\|x-y\|
$$

for $t \in[-\varepsilon, \varepsilon]$. Therefore

$$
\|\mathscr{F} x-\mathscr{F} y\| \leq \beta\|x-y\|, \quad x, y \in \mathscr{B},
$$

where $\beta=2 \varepsilon \sinh \varepsilon<1$ by (2.7). Hence (ii) is satisfied.

Lemma 2.6. Let $\alpha \in(0,1)$. Then

(a) there exists a maximal solution of (1.3) satisfying the condition

$$
y(0)=0, \quad y^{\prime}(0)=\alpha,
$$

(b) there exists a unique $t_{0} \in(0, \infty)$ such that any maximal solution $y(t)$ of the initial value problem (1.3), (2.10) satisfies $y\left(t_{0}\right)=t_{0}, y(t)<t$ for $t \in\left(0, t_{0}\right)$ and

$$
y(t)=-y(-t) \quad \text { for } t \in\left[-t_{0}, 0\right],
$$

(c) there exists a unique solution of the initial value problem (1.3), (2.10) on the interval $\left[-t_{0}, t_{0}\right]$.

Proof. By Lemma 2.5, there exists a unique solution $v$ of (1.3) on an interval $[-\varepsilon, \varepsilon], \varepsilon>0$, satisfying (2.10) (with $y=v$ ). Let $y$ be a maximal solution of $(1.3)$ on an interval $J$ which is a continuation of $v$. Hence (a) is true. From $(2.10)$ we deduce that $y(t)<t$ for $t \in\left(0, \varepsilon_{0}\right)$ with an $\varepsilon_{0} \in(0, \varepsilon]$. Let $J_{1}=\{t: t \in J, 0<y(s)<s$ for $0<s \leq t\}$. Then $y(y(t))<y(t)$ for $t \in J_{1}$, and therefore $y^{\prime \prime}(t)=y(t)-y(y(t))>0$ on this interval. Hence $y^{\prime}$ is increasing and $y^{\prime}>\alpha$ on $J_{1}$ by $(2.10)$. If $J_{1}=(0, \infty)$, then $\lim _{t \rightarrow \infty} y^{\prime}(t) \leq 1$ (see the proof of Lemma 2.3) and from

$$
y^{\prime \prime \prime}(t)=y^{\prime}(t)\left[1-y^{\prime}(y(t))\right]>0, \quad t \in(0, \infty)
$$

and $y^{\prime \prime}(0)=0$ we see that $y^{\prime \prime}$ is increasing and $y^{\prime \prime}>0$ on $(0, \infty)$, which contradicts $\lim _{t \rightarrow \infty} y^{\prime}(t) \leq 1$. Therefore

$$
\sup \left\{t: t \in J_{1}\right\}=t_{0}<\infty, \quad t_{0} \geq \varepsilon_{0} .
$$

We now show that $y\left(t_{0}\right)=t_{0}$. First we prove that $t_{0} \in J$. If not, using the same procedure as in the proof of Lemma 2.3, there is a right continuation of $y$ on $J \cup\left\{t_{0}\right\}$, contrary to $y$ being a maximal solution of (1.3). Hence $t_{0} \in J$. If $y\left(t_{0}\right)<t_{0}$, then using the same procedure as in the proof of Lemma 2.3, there exists $\tau>0$ such that $J_{1} \cup\left(t_{0}, t_{0}+\tau\right] \subset J$ and $y(t)<t$ for $t \in J_{1} \cup\left(t_{0}, t_{0}+\tau\right]$, contrary to the definition of $J_{1}$ and $t_{0}$. Hence $y\left(t_{0}\right)=t_{0}$. Let $u$ be another 
maximal solution of the initial value problem (1.3), (2.10) and let $u\left(t_{1}\right)=t_{1}$ and $u(t)<t$ for $t \in\left(0, t_{1}\right)$ with a $t_{1}>0$. Without loss of generality we can assume that $t_{1} \leq t_{0}$. We now prove that $u(t)=y(t)$ for $t \in\left[0, t_{1}\right]$. Assume $y(t) \not \equiv u(t)$ for $t \in\left[0, t_{1}\right]$. Lemma 2.5 shows that $y(t)=u(t)$ for $t \in[0, \varepsilon]$ with an $\varepsilon>0$. Let

$$
\chi=\max \left\{t: t \in\left[0, t_{1}\right], y(s)=u(s) \text { for } s \in[0, t]\right\}
$$

Set

$$
\varrho(t)=\max \{|y(s)-u(s)|: \chi \leq s \leq t\}, \quad t \in\left[\chi, t_{1}\right],
$$

and $Q=\max \left\{\left|u^{\prime}(t)\right|: 0 \leq t \leq t_{1}\right\}$. Then $\chi \geq \varepsilon, \varrho \in C^{0}\left(\left[\chi, t_{1}\right]\right)$ is nondecreasing, $\varrho(\chi)=0$ and $\varrho>0$ on $\left(\chi, t_{1}\right]$. Since $y(\chi)<\chi$ and $y$ is increasing on $\left[0, t_{0}\right]$, there exists $\mu \in\left(0, t_{1}-\chi\right]$ such that $y(t) \leq \chi$ for $t \in[0, \chi+\mu]$ and then

$$
u(y(t))-y(y(t))=0, \quad t \in[0, \chi+\mu] .
$$

Hence

$$
\begin{aligned}
|u(u(t))-y(y(t))| & =|[u(u(t))-u(y(t))]-[u(y(t))-y(y(t))]| \\
& =|u(u(t))-u(y(t))|=\left|u^{\prime}(\xi)(u(t)-y(t))\right| \\
& \leq Q|u(t)-y(t)|
\end{aligned}
$$

for $t \in[0, \chi+\mu]$ where $\xi$ lies between $u(t)$ and $y(t)$. Since (see Lemma 2.4)

$$
|y(t)-u(t)|=\left|\int_{0}^{t} \sinh (t-s)[u(u(s))-y(y(s))] d s\right|, \quad t \in\left[0, t_{1}\right],
$$

it follows from the properties of $y$ and $u$ and (2.13) that

$$
|y(t)-u(t)| \leq Q \int_{\chi}^{t} \sinh (t-s)|u(s)-y(s)| d s, \quad t \in[\chi, \chi+\mu] .
$$

Hence (see (2.12))

$$
|y(s)-u(s)| \leq Q \sinh \mu \varrho(t)(t-\chi), \quad \chi \leq s \leq t \leq \chi+\mu
$$

and then

$$
\varrho(t) \leq Q \sinh \mu \varrho(t)(t-\chi), \quad t \in[\chi, \chi+\mu] .
$$

Consequently $1 \leq Q \sinh \mu(t-\chi)$ for $t \in(\chi, \chi+\mu]$ since $\varrho>0$ on $\left(\chi, t_{1}\right]$, which is impossible. Summarizing, we have proved the existence of a unique $t_{0} \in(0, \infty)$ such that any maximal solution $y(t)$ of the initial value problem (1.3), (2.10) satisfies $y\left(t_{0}\right)=t_{0}, y(t)<t$ for $t \in\left(0, t_{0}\right)$ and also that the above problem has a unique solution on $\left[0, t_{0}\right]$. 
Let $y(t)$ be a maximal solution of (1.3) on an interval $J$ satisfying (2.10). For (b), it remains to verify (2.11). Similar reasoning as in the first part of our proof shows that there is a $\hat{t}<0$ such that $[\hat{t}, 0] \subset J, y(\hat{t})=\hat{t}$ and $y(t)>t$ for $t \in(\hat{t}, 0)$. Set

$$
w(t)=-y(-t), \quad t \in \hat{J}=\{t:-t \in J\} .
$$

By Lemma 2.1 (with $c=0$ and $\mu=-1$ ), $w$ is a solution on $\hat{J} \supset\left[-t_{0}, 0\right]$. Since $w(0)=0$ and $w^{\prime}(0)=y^{\prime}(0)=\alpha$, using Lemma 2.5, we have $w(t)=y(t)$ for $t \in[-\varepsilon, 0]$. If $\varepsilon \geq t_{0},(2.11)$ is true. Let $\varepsilon<t_{0}$. There is no loss of generality in assuming $\hat{t} \leq-t_{0}$. Suppose that $w(t) \not \equiv y(t)$ on $\left[-t_{0},-\varepsilon\right]$ and let

$$
d=\min \{t: w(s)=y(s) \text { for } s \in[t, 0]\} .
$$

Then $d \in\left(-t_{0},-\varepsilon\right]$ and from the equalities

$$
\begin{aligned}
& y(t)=y(d)+y^{\prime}(d)(t-d)+\int_{d}^{t}(t-s)[y(s)-y(y(s))] d s, \\
& w(t)=w(d)+w^{\prime}(d)(t-d)+\int_{d}^{t}(t-s)[w(s)-w(w(s))] d s
\end{aligned}
$$

for $t \in\left[-t_{0}, d\right]$ and $y(d)=w(d), y^{\prime}(d)=w^{\prime}(d)$, we get

$$
\begin{aligned}
y(t)-w(t)= & \int_{d}^{t}(t-s)[y(s)-w(s)-y(y(s))+w(w(s))] d s, \\
& t \in\left[-t_{0}, d\right] .
\end{aligned}
$$

Since $w(t)>t$ on a neighbourhood of $t=d$, there exists $\varepsilon_{*}>0$ such that $w(t) \geq d$ for $t \in\left[d-\varepsilon_{*}, d\right] \subset\left[-t_{0}, d\right]$. Then $w(w(t))=y(w(t))$ for $t \in\left[d-\varepsilon_{*}, d\right]$, and consequently

$$
\begin{aligned}
y(y(t))-w(w(t)) & =[y(y(t))-y(w(t))]+[y(w(t))-w(w(t))] \\
& =y(y(t))-y(w(t))=y^{\prime}(\xi)(y(t)-w(t))
\end{aligned}
$$

for $t \in\left[d-\varepsilon_{*}, d\right]$ where $\xi$ lies between $y(t)$ and $w(t)$. Hence

$$
|y(y(t))-w(w(t))| \leq S|y(t)-w(t)|, \quad t \in\left[d-\varepsilon_{*}, d\right]
$$

where $S=\max \left\{\left|y^{\prime}(t)\right|: t \in\left[-t_{0}, 0\right]\right\}$. Now (2.14) gives

$$
|y(t)-w(t)| \leq \varepsilon_{*}(1+S) \int_{t}^{d}|y(s)-w(s)| d s, \quad t \in\left[d-\varepsilon_{*}, d\right] .
$$

Let

$$
\gamma(t)=\max \{|y(s)-w(s)|: s \in[t, d]\}, \quad t \in\left[d-\varepsilon_{*}, d\right] .
$$


Then $\gamma \in C^{0}\left(\left[d-\varepsilon_{*}, d\right]\right)$ is nonincreasing, $\gamma(d)=0$ and $\gamma>0$ on $\left[d-\varepsilon_{*}, d\right)$. From (2.15) we now deduce that

$$
|y(s)-w(s)| \leq \varepsilon_{*}(1+S) \gamma(t)(d-t), \quad d-\varepsilon_{*} \leq t \leq s \leq d .
$$

Therefore

$$
\gamma(t) \leq \varepsilon_{*}(1+S) \gamma(t)(d-t), \quad t \in\left[d-\varepsilon_{*}, d\right]
$$

and $1 \leq \varepsilon_{*}(1+S)(d-t)$ for $t \in\left[d-\varepsilon_{*}, d\right)$, which is impossible. We have proved that $w(t)=y(t)$ for $t \in\left[-t_{0}, 0\right]$. Therefore (b) is true.

Since we have verified that the initial value problem (1.3), (2.10) has a unique solution on $\left[0, t_{0}\right]$, the validity of (c) follows now immediately from (b).

Lemma 2.7. Let $y(t)$ be a maximal solution of (1.3) on an interval $J$, $y(0)=0$ and $y^{\prime}(0)=1$. Then $J=\boldsymbol{R}$ and $y(t)=t$ for $t \in \boldsymbol{R}$.

Proof. The function $u(t)=t$ for $t \in \boldsymbol{R}$ is a maximal solution of (1.3) on $\boldsymbol{R}$ satisfying $u(0)=0, u^{\prime}(0)=1$. In order to prove our lemma we have to show that $y=u$. Assume on the contrary that $y \neq u$ and let $y(t) \not \equiv u(t)$ for $t \in[0, \infty) \cap J$ (the case when $y(t) \not \equiv u(t)$ for $t \in(-\infty, 0] \cap J$ is treated in a similar fashion). Then there is an $\varepsilon \geq 0$ such that $y(t)=u(t)$ for $t \in[0, \varepsilon]$ and the function $\delta:[\varepsilon, \infty) \cap J \rightarrow \boldsymbol{R}$ defined by

$$
\delta(t)=\max \{|y(s)-s|: s \in[\varepsilon, t]\}
$$

is continuous and nondecreasing on $[\varepsilon, \infty) \cap J, \delta(\varepsilon)=0$ and $\delta(t)>0$ for $t \in(\varepsilon, \infty) \cap J$. Let $\gamma=\sup \{t: t \in J\}$. Now from the equality

$$
y(t)=t+\int_{\varepsilon}^{t}(t-s)[y(s)-y(y(s))] d s, \quad t \in[\varepsilon, \infty) \cap J
$$

we deduce that

$$
|y(t)-t|=\int_{\varepsilon}^{t}(t-s)\left|y^{\prime}(\eta)\right||y(s)-s| d s \leq(t-\varepsilon) V \int_{\varepsilon}^{t}|y(s)-s| d s
$$

for $t \in[\varepsilon, \varepsilon+v]$ where $v=\min \{1,(\gamma-\varepsilon) / 2\}, \eta$ lies between $s$ and $y(s)$ and

$$
V=\max \left\{\left|y^{\prime}(t)\right|: \varepsilon \leq t \leq \max \left\{\varepsilon+v, \max _{s \in[\varepsilon, \varepsilon+v]} y(s)\right\}\right\} .
$$

From (2.16) and (2.17) it follows that $\delta(t) \leq(t-\varepsilon)^{2} V \delta(t)$ for $t \in[\varepsilon, \varepsilon+v]$ and then $1 \leq(t-\varepsilon)^{2} V$ for $t \in(\varepsilon, \varepsilon+v]$, which is impossible. Hence $J=\boldsymbol{R}$ and $y(t)=t$. 
Lemma 2.8. Let $y(t)$ be a maximal solution of (1.3) on an interval $J$ and let

$$
y(0)=0, \quad y^{\prime}(0)>1 .
$$

Then there exists $t_{0} \in(0, \infty) \cap J$ such that $y\left(t_{0}\right)=t_{0}, y(t)>t$ for $t \in\left(0, t_{0}\right)$ and $y^{\prime}\left(t_{0}\right) \in(0,1)$.

Proof. (2.18) implies that $y(t)>t$ on a right neighbourhood of $t=0$. Assume that $y(t)>t$ for $t \in J_{1}=(0, \infty) \cap J$. If $\sup \left\{t: t \in J_{1}\right\}=a<\infty$ then, by Lemma 2.1, $t<y(t) \leq a$ for $t \in J_{1}$, therefore $\lim _{t \rightarrow a^{-}} y(t)=a$ and $y(t)>t$ for $t \in J_{1}$ implies $a \notin J_{1}$. Then we can verify (see the proof of Lemma 2.3) that the function $z: J \cup\{a\} \rightarrow \boldsymbol{R}$,

$$
z(t)= \begin{cases}y(t) & \text { for } t \in J \\ a & \text { for } t=a\end{cases}
$$

is a solution of (1.3) on $J \cup\{a\}$, and so $z$ is a right continuation of $y$, contrary to $y$ being a maximal solution of $(1.3)$. Hence $J_{1}=(0, \infty)$ and then $y(y(t))-$ $y(t)>0$ for $t \in J_{1}$ implies $y^{\prime \prime}<0$ on $J_{1}$. Therefore $y^{\prime}$ is decreasing on $J_{1}$ and $y(t)>t$ gives $y^{\prime}>1$ on $J_{1}$. Then $y^{\prime \prime \prime}(t)=y^{\prime}(t)\left(1-y^{\prime}(y(t))\right)<0$ and $y^{\prime \prime} \leq-K$ on an interval $\left[t_{*}, \infty\right), t_{*}>0$, with a positive constant $K$, contrary to $y^{\prime}>1$ on $J_{1}$. It follows that $y\left(t_{0}\right)=t_{0}$ for a $t_{0}>0$ and $y(t)>t$ for $t \in\left(0, t_{0}\right)$. Now $y^{\prime \prime}<0$ on $\left(0, t_{0}\right)$ implies that $y^{\prime}$ is decreasing on $\left[0, t_{0}\right]$ and then $t_{0}=y\left(t_{0}\right)=$ $y\left(t_{0}\right)-y(0)=y^{\prime}(\xi) t_{0}$ with a $\xi \in\left(0, t_{0}\right)$ gives $y^{\prime}(\xi)=1$. Hence $y^{\prime}\left(t_{0}\right)<y^{\prime}(\xi)$ $=1$ and $y^{\prime}\left(t_{0}\right) \in(0,1)$.

\section{The structure of the set $\Omega$ and properties of the function $T(\alpha)$}

We first show that for any $\alpha \in(0,1)$ there exists a unique element $y$ in the set $\Omega$ defined in Section 1 satisfying (2.10).

Lemma 3.1. For each $\alpha \in(0,1)$ there exists a unique $y \in \Omega$ satisfying (2.10). In addition, $y(-t)=-y(t)$ for $t \in \boldsymbol{R}$.

Proof. Fix $\alpha \in(0,1)$. By Lemma 2.6, there is a unique solution $u$ of (1.3) on an interval $\left[-t_{0}, t_{0}\right], t_{0}>0$, such that $u(0)=0, u^{\prime}(0)=\alpha, u\left(t_{0}\right)=t_{0}, u(t)<t$ for $t \in\left(0, t_{0}\right)$ and $u(t)=-u(-t)$ for $t \in\left[-t_{0}, 0\right]$. Then $u\left(-t_{0}\right)=-u\left(t_{0}\right)=-t_{0}$, $u^{\prime}\left(-t_{0}\right)=u^{\prime}\left(t_{0}\right)$ and $u^{\prime \prime}\left(-t_{0}\right)=-u^{\prime \prime}\left(t_{0}\right)=0$. Also $u^{\prime}>0$ on $\left[-t_{0}, t_{0}\right]$ since $u^{\prime}$ is decreasing on $\left[-t_{0}, 0\right]$ and increasing on $\left[0, t_{0}\right]$. Let $w:\left[-t_{0}, t_{0}\right] \rightarrow \boldsymbol{R}$ be the function defined by

$$
w(t)=t-u(t) .
$$

Then $w\left(-t_{0}\right)=w\left(t_{0}\right)=0, w^{\prime}\left(-t_{0}\right)=w^{\prime}\left(t_{0}\right)=1-u^{\prime}\left(t_{0}\right), \quad w^{\prime \prime}\left(-t_{0}\right)=w^{\prime \prime}\left(t_{0}\right)=0$ and $w^{\prime \prime}(t)=-w(t-w(t))$ for $t \in\left[-t_{0}, t_{0}\right]$. Write $w_{*}$ for the $2 t_{0}$-periodic continuation of $w$ on $\boldsymbol{R}$ and set 


$$
y(t)=t-w_{*}(t), \quad t \in \boldsymbol{R} .
$$

Then $y(-t)=-y(t)$ for $t \in \boldsymbol{R}, t-y(t)$ is a $2 t_{0}$-periodic function, $y^{\prime}>0$ on $\boldsymbol{R}$ since $y^{\prime}$ is $2 t_{0}$-periodic and $y^{\prime}(t)=u^{\prime}(t)>0$ for $t \in\left[-t_{0}, t_{0}\right]$, and from $w_{*}^{\prime \prime}(t)=$ $-w_{*}\left(t-w_{*}(t)\right)$ for $t \in \boldsymbol{R}$, we have

$$
y^{\prime \prime}(t)=-w_{*}^{\prime \prime}(t)=w_{*}\left(t-w_{*}(t)\right)=w_{*}(y(t))=y(t)-y(y(t)), \quad t \in \boldsymbol{R} .
$$

Hence $y$ is the unique element of $\Omega$ satisfying (2.10).

For each $\alpha \in(0,1)$, denote by $y_{\alpha}$ the unique element of $\Omega$ satisfying (2.10). The existence and uniqueness of $y_{\alpha}$ is guaranteed by Lemma 3.1. Next Lemma 2.6 shows that for each $y_{\alpha}$ with $\alpha \in(0,1)$, there exists a unique $T(\alpha)>0$ such that

$$
y_{\alpha}(T(\alpha))=T(\alpha) \quad \text { and } \quad y_{\alpha}(t)<t \text { for } t \in(0, T(\alpha)) .
$$

Thus we obtain the function $T:(0,1) \rightarrow(0, \infty)$. Using this function $T$, we can describe the period of $y_{\alpha}(t)-t$, namely the number $2 T(\alpha)$ is its period.

We also note that $y(t) \equiv t$ is a unique solution of problem (1.3), (1.4) on $\boldsymbol{R}$ satisfying (2.10) with $\alpha=1$ by Lemma 2.7 , but $y \notin \Omega$ which follows from the definition of the set $\Omega$.

In the next lemma, we describe all elements of the set $\Omega$ by $y_{\alpha}$ with $\alpha \in(0,1)$.

Lemma 3.2. Let $\Gamma=\left\{c+y_{\alpha}(t-c): c \in \boldsymbol{R}, \alpha \in(0,1)\right\}$. Then $\Omega=\Gamma$.

Proof. We know that $y_{\alpha} \in \Omega$ for each $\alpha \in(0,1)$. Next Lemma 2.2 implies that $c+y_{\alpha}(t-c)$ is a solution of (1.3) on $\boldsymbol{R}$ for each $c \in \boldsymbol{R}$. Since $\left(c+y_{\alpha}(t-c)\right)^{\prime}=y_{\alpha}^{\prime}(t-c)>0$ for $t \in \boldsymbol{R}$ and $y_{\alpha}(t-c)+c-t$ is a periodic function, $c+y_{\alpha}(t-c) \in \Omega$ for each $c \in \boldsymbol{R}$ and $\alpha \in(0,1)$. Hence $\Gamma \subset \Omega$.

Let now $u \in \Omega$. Then $u\left(t_{0}\right)=t_{0}$ with a $t_{0} \in \boldsymbol{R}$ by Lemma 2.3. Setting $u_{0}(t)=t_{0}-u\left(t_{0}-t\right)$ for $t \in \boldsymbol{R}, u_{0}$ is a solution of (1.3) on $\boldsymbol{R}$ (see Lemma 2.2), $u_{0}(0)=0, u_{0}^{\prime}>0$ on $\boldsymbol{R}$ and $u_{0}(t)-t$ is periodic. If $u_{0}^{\prime}(0)=\alpha \in(0,1)$, then $u_{0}=y_{\alpha}$ by Lemma 2.6, and since $y_{\alpha}$ is an odd function, we have

$$
u(t)=t_{0}-u_{0}\left(t_{0}-t\right)=t_{0}-y_{\alpha}\left(t_{0}-t\right)=t_{0}+y_{\alpha}\left(t-t_{0}\right), \quad t \in \boldsymbol{R} .
$$

Hence $u \in \Gamma$. Let $u_{0}^{\prime}(0)>1$. Then Lemma 2.8 guarantees that $u_{0}\left(t_{*}\right)=t_{*}$ with a $t_{*}>0, u_{0}(t)>t$ for $t \in\left(0, t_{*}\right)$ and $u_{0}^{\prime}\left(t_{*}\right)=\beta \in(0,1)$. Set

$$
\hat{u}(t)=t_{*}-u_{0}\left(t_{*}-t\right), \quad t \in \boldsymbol{R} .
$$

Then $\hat{u}$ is a solution of (1.3) on $\boldsymbol{R}$ by Lemma 2.2 (with $c=t_{*}$ and $\mu=-1$ ), $\hat{u}^{\prime}>0$ on $\boldsymbol{R}, \hat{u}(t)-t$ is periodic and $\hat{u}(0)=0, \hat{u}^{\prime}(0)=u_{0}^{\prime}\left(t_{*}\right)=\beta$. Hence $\hat{u}=y_{\beta}$ and then 


$$
u(t)=t_{0}-u_{0}\left(t_{0}-t\right)=t_{0}-t_{*}+y_{\beta}\left(t-t_{0}+t_{*}\right), \quad t \in \boldsymbol{R} .
$$

Therefore $u \in \Gamma$. We have verified that $\Omega \subset \Gamma$. Consequently, $\Omega=\Gamma$.

Lemma 3.3. The period $2 T(\alpha)$ of $y_{\alpha}(t), \alpha \in(0,1)$, satisfies the inequality

$$
\ln \frac{1}{\alpha^{2}}<2 T(\alpha)<\frac{2 \pi}{\sqrt{\alpha}} .
$$

Proof. Since $y_{\alpha}(0)=0, y_{\alpha}^{\prime}(0)=\alpha$ and $y_{\alpha}^{\prime}$ is increasing on $[0, T(\alpha)]$, integrating the equality

$$
2 y_{\alpha}^{\prime}(t) y_{\alpha}^{\prime \prime}(t)=2 y_{\alpha}(t) y_{\alpha}^{\prime}(t)-2 y_{\alpha}\left(y_{\alpha}(t)\right) y_{\alpha}^{\prime}(t)
$$

from 0 to $t \in(0, T(\alpha)]$, we get

$$
y_{\alpha}^{\prime 2}(t)=\alpha^{2}+y_{\alpha}^{2}(t)-2 \int_{0}^{t} y_{\alpha}\left(y_{\alpha}(s)\right) y_{\alpha}^{\prime}(s) d s, \quad t \in[0, T(\alpha)] .
$$

Then

$$
y_{\alpha}^{\prime 2}(t)<\alpha^{2}+y_{\alpha}^{2}(t)<\left(\alpha+y_{\alpha}(t)\right)^{2}, \quad t \in(0, T(\alpha)]
$$

and from the inequality $y_{\alpha}^{\prime}(t)<\alpha+y_{\alpha}(t)$ we deduce that

$$
y_{\alpha}(t)<\alpha\left(e^{t}-1\right), \quad t \in(0, T(\alpha)] .
$$

Consider the function $p(t)=\alpha\left(e^{t}-1\right)-t$ on the interval $[0, \infty)$. Since $p(0)=0$, $p^{\prime}(0)=\alpha-1<0, \lim _{t \rightarrow \infty} p(t)=\infty$ and $p^{\prime}(\xi)=\alpha e^{\xi}-1=0$ if and only if $\xi=\ln (1 / \alpha)$, we see that there exists a unique $t_{*}>0$ such that $p\left(t_{*}\right)=0$ and $t_{*}>\xi=\ln (1 / \alpha)$. Now (3.3) gives $y_{\alpha}(t)<p(t)+t \leq t_{*}$ for $t \in\left(0, t_{*}\right]$, and therefore $t_{*}<T(\alpha)$ which proves that $2 T(\alpha)>2 t_{*}>\ln \left(1 / \alpha^{2}\right)$.

It remains to prove $T(\alpha)<\pi / \sqrt{\alpha}$. Since $y_{\alpha}^{\prime}$ is increasing on $[0, T(\alpha)]$ and $y_{\alpha}^{\prime}(0)=\alpha$, we have $y_{\alpha}^{\prime}>\alpha$ on $(0, T(\alpha)]$ and then

$$
y_{\alpha}^{\prime \prime}(t)=y_{\alpha}(t)-y_{\alpha}\left(y_{\alpha}(t)\right)=y_{\alpha}^{\prime}(\eta)\left(t-y_{\alpha}(t)\right)>\alpha\left(t-y_{\alpha}(t)\right)
$$

for $t \in(0, T(\alpha)]$ where $\eta \in\left(y_{\alpha}(t), t\right)$. Set

$$
w(t)=t-y_{\alpha}(t), \quad t \in[0, T(\alpha)] .
$$

Then $w(0)=w(T(\alpha))=0, w^{\prime}(0)=1-\alpha, w>0$ on $(0, T(\alpha))$ and (see (3.4)) $w^{\prime \prime}(t)=-y_{\alpha}^{\prime \prime}(t)<-\alpha w(t)$ for $t \in(0, T(\alpha)]$. Hence $w^{\prime \prime}(t)=-\alpha w(t)+q(t)$ for $t \in[0, T(\alpha)]$ where $q \in C^{0}([0, T(\alpha)])$ is a negative function on $(0, T(\alpha)]$, and so

$$
w(t)=\frac{1-\alpha}{\sqrt{\alpha}} \sin (\sqrt{\alpha} t)+\frac{1}{\sqrt{\alpha}} \int_{0}^{t} \sin (\sqrt{\alpha}(t-s)) q(s) d s, \quad t \in[0, T(\alpha)]
$$


Since

$$
\int_{0}^{\pi / \sqrt{\alpha}} \sin \left(\sqrt{\alpha}\left(\frac{\pi}{\sqrt{\alpha}}-s\right)\right) q(s) d s<0
$$

and then (see (3.5))

$$
w\left(\frac{\pi}{\sqrt{\alpha}}\right)=\frac{1}{\sqrt{\alpha}} \int_{0}^{\pi / \sqrt{\alpha}} \sin \left(\sqrt{\alpha}\left(\frac{\pi}{\sqrt{\alpha}}-s\right)\right) q(s) d s<0,
$$

we deduce from the inequality $w(t) \geq 0$ on $[0, T(\alpha)]$ that $T(\alpha)<\pi / \sqrt{\alpha}$, which completes the proof.

Lemma 3.4. The function $T(\alpha)$ is continuous on $(0,1)$ and

$$
\lim _{\alpha \rightarrow 0^{+}} T(\alpha)=\infty, \quad \lim _{\alpha \rightarrow 1^{-}} T(\alpha) \leq \pi
$$

Proof. The continuity of $T(\alpha)$ on $(0,1)$ is proved by a way of contradiction. Assume that $T(\alpha)$ is discontinuous at a point $\alpha=\alpha_{0}, \alpha_{0} \in(0,1)$. Then there exist $\varepsilon_{0}>0$ and a sequence $\left\{\alpha_{n}\right\} \subset\left(\alpha_{0} / 2,\left(1+\alpha_{0}\right) / 2\right), \lim _{n \rightarrow \infty} \alpha_{n}=\alpha_{0}$ such that

$$
\left|T\left(\alpha_{n}\right)-T\left(\alpha_{0}\right)\right| \geq \varepsilon_{0} \quad \text { for } n \in N .
$$

The next part of the proof is divided into two cases.

Case (i). There is a subsequence $\left\{\alpha_{k_{n}}\right\}$ of $\left\{\alpha_{n}\right\}$ such that

$$
T\left(\alpha_{k_{n}}\right) \geq T\left(\alpha_{0}\right)+\varepsilon_{0} \quad \text { for } n \in N \text {. }
$$

From (3.2) and $\alpha_{k_{n}}>\alpha_{0} / 2$ we obtain

$$
T\left(\alpha_{k_{n}}\right)<\frac{\pi}{\sqrt{\alpha_{k_{n}}}}<\sqrt{\frac{2}{\alpha_{0}}} \pi, \quad n \in N,
$$

and consequently $\left\{T\left(\alpha_{k_{n}}\right)\right\}$ is bounded. Hence we can assume that $\left\{T\left(\alpha_{k_{n}}\right)\right\}$ is convergent and let $\lim _{n \rightarrow \infty} T\left(\alpha_{k_{n}}\right)=\delta$. Then (see (3.8))

$$
\delta \geq T\left(\alpha_{0}\right)+\varepsilon_{0}
$$

As

$0<y_{\alpha_{k_{n}}}(t)<t, \quad 0<y_{\alpha_{k_{n}}}\left(y_{\alpha_{k_{n}}}(t)\right)<t, \quad 0<y_{\alpha_{k_{n}}}^{\prime \prime}(t)=y_{\alpha_{k_{n}}}(t)-y_{\alpha_{k_{n}}}\left(y_{\alpha_{k_{n}}}(t)\right)<t$ for $t \in\left(0, T\left(\alpha_{0}\right)\right]$ and $n \in N,\left\{y_{\alpha_{k_{n}}}^{\prime}(t)\right\}$ is equicontinuous on $\left[0, T\left(\alpha_{0}\right)\right]$ and since $y_{\alpha_{k_{n}}}^{\prime}(0)=\alpha_{k_{n}},\left\{y_{\alpha_{k_{n}}}\right\}$ is bounded in $C^{1}\left(\left[0, T\left(\alpha_{0}\right)\right]\right)$. Applying the Arzelà-Ascoli theorem we can assume without loss of generality that $\left\{y_{\alpha_{k_{n}}}\right\}$ is convergent in $C^{1}\left(\left[0, T\left(\alpha_{0}\right)\right]\right)$ and let $\lim _{n \rightarrow \infty} y_{\alpha_{k_{n}}}=\beta$. Then from the equalities 


$$
\begin{aligned}
y_{\alpha_{k_{n}}}\left(y_{\alpha_{k_{n}}}(t)\right)-\beta(\beta(t)) & =\left[y_{\alpha_{k_{n}}}\left(y_{\alpha_{k_{n}}}(t)\right)-y_{\alpha_{k_{n}}}(\beta(t))\right]+\left[y_{\alpha_{k_{n}}}(\beta(t))-\beta(\beta(t))\right] \\
& =y_{\alpha_{k_{n}}}^{\prime}(\xi)\left(y_{\alpha_{k_{n}}}(t)-\beta(t)\right)+\left[y_{\alpha_{k_{n}}}(\beta(t))-\beta(\beta(t))\right]
\end{aligned}
$$

where $\xi$ lies between $y_{\alpha_{k_{n}}}(t)$ and $\beta(t)$, we deduce that $\lim _{n \rightarrow \infty} y_{\alpha_{k_{n}}}\left(y_{\alpha_{k_{n}}}(t)\right)=$ $\beta(\beta(t))$ uniformly on $\left[0, T\left(\alpha_{0}\right)\right]$. Letting $n \rightarrow \infty$ in the equality (for $t \in\left[0, T\left(\alpha_{0}\right)\right]$ and $n \in N$ )

$$
y_{\alpha_{k_{n}}}(t)=\alpha_{k_{n}} t+\int_{0}^{t}(t-s)\left[y_{\alpha_{k_{n}}}(s)-y_{\alpha_{k_{n}}}\left(y_{\alpha_{k_{n}}}(s)\right)\right] d s,
$$

we have

$$
\beta(t)=\alpha_{0} t+\int_{0}^{t}(t-s)[\beta(s)-\beta(\beta(s))] d s, \quad t \in\left[0, T\left(\alpha_{0}\right)\right] .
$$

Hence $\beta(0)=0, \beta^{\prime}(0)=\alpha_{0}$ and $\beta^{\prime \prime}(t)=\beta(t)-\beta(\beta(t))$ for $t \in\left[0, T\left(\alpha_{0}\right)\right]$, and therefore $\beta(t)=y_{\alpha_{0}}(t)$ for $t \in\left[0, T\left(\alpha_{0}\right)\right]$ by Lemma 2.6. We have proved $\lim _{n \rightarrow \infty} y_{\alpha_{k_{n}}}^{(j)}(t)=y_{\alpha_{0}}^{(j)}(t)$ uniformly on $\left[0, T\left(\alpha_{0}\right)\right]$ for $j=0,1$. Then

$$
\lim _{n \rightarrow \infty} y_{\alpha_{k_{n}}}\left(T\left(\alpha_{0}\right)\right)=y_{\alpha_{0}}\left(T\left(\alpha_{0}\right)\right)=T\left(\alpha_{0}\right) .
$$

From $\quad T\left(\alpha_{0}\right)=y_{\alpha_{0}}\left(T\left(\alpha_{0}\right)\right)=y_{\alpha_{0}}\left(T\left(\alpha_{0}\right)\right)-y_{\alpha_{0}}(0)=y_{\alpha_{0}}^{\prime}(\eta) T\left(\alpha_{0}\right) \quad$ where $\quad \eta \in$ $\left(0, T\left(\alpha_{0}\right)\right)$, it follows that $y_{\alpha_{0}}^{\prime}(\eta)=1$. Since $0<y_{\alpha_{0}}(t)<t$ for $t \in\left(0, T\left(\alpha_{0}\right)\right)$, we have $y_{\alpha_{0}}^{\prime \prime}(t)=y_{\alpha_{0}}(t)-y_{\alpha_{0}}\left(y_{\alpha_{0}}(t)\right)>0$ on this interval. Hence $y_{\alpha_{0}}^{\prime}$ is increasing on $\left(0, T\left(\alpha_{0}\right)\right)$ and $y_{\alpha_{0}}^{\prime}(\eta)=1$ shows that $y_{\alpha_{0}}^{\prime}\left(T\left(\alpha_{0}\right)\right)>1$. Now the equality $\lim _{n \rightarrow \infty} y_{\alpha_{k_{n}}}^{\prime}\left(T\left(\alpha_{0}\right)\right)=y_{\alpha_{0}}^{\prime}\left(T\left(\alpha_{0}\right)\right)$ implies

$$
y_{\alpha_{k_{n}}}^{\prime}\left(T\left(\alpha_{0}\right)\right) \geq \frac{1+y_{\alpha_{0}}^{\prime}\left(T\left(\alpha_{0}\right)\right)}{2}(>1)
$$

for $n \geq n_{0}$ with an $n_{0} \in N$. We know that $y_{\alpha_{k_{n}}}^{\prime}$ is increasing on $\left[0, T\left(\alpha_{k_{n}}\right)\right]$, and therefore

$$
y_{\alpha_{k_{n}}}^{\prime}(t) \geq \frac{1+y_{\alpha_{0}}^{\prime}\left(T\left(\alpha_{0}\right)\right)}{2}, \quad t \in\left[T\left(\alpha_{0}\right), T\left(\alpha_{k_{n}}\right)\right], n \geq n_{0} .
$$

Then (for $n \geq n_{0}$ )

$$
\begin{aligned}
T\left(\alpha_{k_{n}}\right)-y_{\alpha_{k_{n}}}\left(T\left(\alpha_{0}\right)\right) & =y_{\alpha_{k_{n}}}\left(T\left(\alpha_{k_{n}}\right)\right)-y_{\alpha_{k_{n}}}\left(T\left(\alpha_{0}\right)\right) \\
& =y_{\alpha_{k_{n}}}^{\prime}(\eta)\left(T\left(\alpha_{k_{n}}\right)-T\left(\alpha_{0}\right)\right) \\
& \geq \frac{1+y_{\alpha_{0}}^{\prime}\left(T\left(\alpha_{0}\right)\right)}{2}\left(T\left(\alpha_{k_{n}}\right)-T\left(\alpha_{0}\right)\right)
\end{aligned}
$$

where $\eta \in\left(T\left(\alpha_{0}\right), T\left(\alpha_{k_{n}}\right)\right)$. Letting $n \rightarrow \infty$ in (3.13) and using $\lim _{n \rightarrow \infty} T\left(\alpha_{k_{n}}\right)=$ $\delta$, (3.9) and (3.11), we get 


$$
1 \geq \frac{1+y_{\alpha_{0}}^{\prime}\left(T\left(\alpha_{0}\right)\right)}{2}
$$

contrary to $y_{\alpha_{0}}^{\prime}\left(T\left(\alpha_{0}\right)\right)>1$ (see $\left.(3.12)\right)$.

Case (ii). There is a subsequence $\left\{\alpha_{l_{n}}\right\}$ of $\left\{\alpha_{n}\right\}$ such that

$$
T\left(\alpha_{l_{n}}\right) \leq T\left(\alpha_{0}\right)-\varepsilon_{0} \quad \text { for } n \in N
$$

Then (for $n \in \boldsymbol{N}$ )

$$
\begin{gathered}
0<y_{\alpha_{l_{n}}}(t)<3 T\left(\alpha_{0}\right), \quad t \in\left[0,2 T\left(\alpha_{0}\right)\right], \\
0<y_{\alpha_{l n}}(t)<2 T\left(\alpha_{0}\right), \quad t \in\left[0, T\left(\alpha_{0}\right)\right]
\end{gathered}
$$

and

$$
\left|y_{\alpha_{l_{n}}}^{\prime \prime}(t)\right| \leq T\left(\alpha_{l_{n}}\right)<T\left(\alpha_{0}\right), \quad t \in \boldsymbol{R} .
$$

Since $y_{\alpha_{l_{n}}}^{\prime}(0)=\alpha_{l_{n}}$, applying the Arzelà-Ascoli theorem, we can assume (taking a subsequence if necessary) that $\left\{y_{\alpha_{l_{n}}}\right\}$ is convergent in $C^{1}\left(\left[0,2 T\left(\alpha_{0}\right)\right]\right)$ and let $\lim _{n \rightarrow \infty} y_{\alpha_{l_{n}}}=\gamma$. From

$$
y_{\alpha_{l_{n}}}\left(y_{\alpha_{l_{n}}}(t)\right)-\gamma(\gamma(t))=y_{\alpha_{l_{n}}}^{\prime}(\tau)\left(y_{\alpha_{l_{n}}}(t)-\gamma(t)\right)+y_{\alpha_{l_{n}}}(\gamma(t))-\gamma(\gamma(t))
$$

where $\tau$ lies between $y_{\alpha_{l_{n}}}(t)$ and $\gamma(t)$, it follows that $\lim _{n \rightarrow \infty} y_{\alpha_{l_{n}}}\left(y_{\alpha_{l_{n}}}(t)\right)=\gamma(\gamma(t))$ uniformly on $\left[0, T\left(\alpha_{0}\right)\right]$. Taking the limit in (3.10) (with $y_{\alpha_{l_{n}}}$ and $\alpha_{l_{n}}$ instead of $y_{\alpha_{k_{n}}}$ and $\alpha_{k_{n}}$, respectively) as $n \rightarrow \infty$, we have

$$
\gamma(t)=\alpha_{0} t+\int_{0}^{t}(t-s)(\gamma(t)-\gamma(\gamma(t))) d s, \quad t \in\left[0, T\left(\alpha_{0}\right)\right]
$$

Using the same procedure as in Case (i), $\gamma(t)=y_{\alpha_{0}}(t)$ for $t \in\left[0, T\left(\alpha_{0}\right)\right]$, and consequently $\lim _{n \rightarrow \infty} y_{\alpha_{l_{n}}}^{(j)}(t)=y_{\alpha_{0}}^{(j)}(t)$ uniformly on $\left[0, T\left(\alpha_{0}\right)\right]$ for $j=0,1$. Now using the inequality (see (3.2))

$$
T\left(\alpha_{l_{n}}\right)>\ln \frac{1}{\alpha_{l_{n}}}>\ln \frac{2}{1+\alpha_{0}},
$$

and $y_{\alpha_{0}}(t)<t$ on $\left(0, T\left(\alpha_{0}\right)\right)$, we see that

$$
y_{\alpha_{0}}(t)<t-v \quad \text { for } t \in\left[\ln \frac{2}{1+\alpha_{0}}, T\left(\alpha_{0}\right)-\varepsilon_{0}\right]
$$

with a positive constant $v$. In addition, since $\lim _{n \rightarrow \infty} y_{\alpha_{l_{n}}}(t)=y_{\alpha_{0}}(t)$ uniformly on $\left[0, T\left(\alpha_{0}\right)\right]$, there exists $n_{*} \in N$ such that

$$
\left|y_{\alpha_{l n}}(t)-y_{\alpha_{0}}(t)\right|<\frac{v}{2} \quad \text { for } t \in\left[\ln \frac{2}{1+\alpha_{0}}, T\left(\alpha_{0}\right)-\varepsilon_{0}\right], n \geq n_{*} .
$$


Then (see (3.15) and (3.16))

$$
\begin{aligned}
& \frac{v}{2}>y_{\alpha_{l_{n}}}\left(T\left(\alpha_{l_{n}}\right)\right)-y_{\alpha_{0}}\left(T\left(\alpha_{l_{n}}\right)\right)=T\left(\alpha_{l_{n}}\right)-y_{\alpha_{0}}\left(T\left(\alpha_{l_{n}}\right)\right) \\
& \quad>T\left(\alpha_{l_{n}}\right)-T\left(\alpha_{l_{n}}\right)+v=v
\end{aligned}
$$

which is impossible.

We have proved that $T(\alpha)$ is continuous on $(0,1)$. From the inequality (3.2) we deduce the validity of (3.6).

\section{Periodic solutions of problem (1.1), (1.2)}

In Section 1, the set of all periodic solutions of problem (1.1), (1.2) is denoted by $\mathscr{A}_{k}$. The following theorem describes the structure of the set $\mathscr{A}_{k}$ by means of solutions $y_{\alpha}$ to problem (1.3), (1.4) with $\alpha \in(0,1)$. Notice that the functions $y_{\alpha}$ are introduced in Section 3 .

Theorem 4.1. For each $k \in(0, \infty)$,

$$
\mathscr{A}_{k}=\left\{\frac{1}{k}\left(t-c-y_{\alpha}(t-c)\right): c \in \boldsymbol{R}, \alpha \in(0,1)\right\} .
$$

Proof. Fix $k \in(0, \infty)$. By Lemma 3.2, $\Omega=\left\{c+y_{\alpha}(t-c): c \in \boldsymbol{R}\right.$, $\alpha \in(0,1)\}$ is the set of all solutions $y(t)$ of problem (1.3), (1.4) on $\boldsymbol{R}$ for which $x(t)=t-y(t)$ is periodic. The assertion of our theorem now follows from Lemma 1.1 which guarantees that $x \in \mathscr{A}_{k}$ if and only if $x(t)=(t-y(t)) / k$ for $t \in \boldsymbol{R}$ where $y \in \Omega$.

Corollary 4.2. For each $k \in(0, \infty)$ and each $T_{*} \in(2 \pi, \infty)$, there exists a $T_{*}$-periodic solution of problem (1.1), (1.2).

Proof. Fix $k \in(0, \infty)$ and $T_{*} \in(2 \pi, \infty)$. As the function $T(\alpha)$ defined in (3.1) is continuous by Lemma 3.4 and satisfies (3.6), there exists $\alpha_{*} \in(0,1)$ such that $T\left(\alpha_{*}\right)=T_{*} / 2$. Then $T_{*}$ is the period of the function $t-y_{\alpha_{*}}(t)$ and since $\left(t-y_{\alpha_{*}}(t)\right) / k \in \mathscr{A}_{k}$ by Theorem 4.1, $T_{*}$ is the period of the periodic solution $\left(t-y_{\alpha_{*}}(t)\right) / k$ to problem (1.1), (1.2).

Corollary 4.3. For each $k \in(0, \infty)$ and each $a \in(0,1 / k)$, there exists a unique periodic solution $x(t)$ of problem (1.1), (1.2) such that $x(0)=0, x^{\prime}(0)=a$.

Proof. Fix $k \in(0, \infty)$ and $a \in(0,1 / k)$. Set $\alpha_{*}=1-a k \in(0,1)$ and $x(t)=\left(t-y_{\alpha_{*}}(t)\right) / k$ for $t \in \boldsymbol{R}$. Then $x \in \mathscr{A}_{k}$ by Theorem 4.1 and $x(0)=0$, $x^{\prime}(0)=\left(1-y_{\alpha_{*}}^{\prime}(0)\right) / k=\left(1-\alpha_{*}\right) / k=a$. Hence $x$ is a periodic solution of problem (1.1), (1.2) and $x(0)=0, x^{\prime}(0)=a$. In order to prove the uniqueness of $x$ with the above properties assume that $u$ is another solution, $u \neq x$. Then, by Theorem 4.1 , there exist $c_{0} \in \boldsymbol{R}$ and $\alpha_{0} \in(0,1)$ such that 


$$
u(t)=\frac{1}{k}\left(t-c_{0}-y_{\alpha_{0}}\left(t-c_{0}\right)\right), \quad t \in \boldsymbol{R} .
$$

Now $u(0)=0, u^{\prime}(0)=a$ imply $y_{\alpha_{0}}\left(-c_{0}\right)=-c_{0}$ and $y_{\alpha_{0}}^{\prime}\left(-c_{0}\right)=1-a k=\alpha_{*}$. From these equalities we deduce that $-c_{0}=2 l T\left(\alpha_{0}\right)$ where $l$ is an integer and $\alpha_{0}=\alpha_{*}$. Since $2 T\left(\alpha_{*}\right)$ is the period of the function $t-y_{\alpha_{*}}(t)$, we have

$$
u(t)=\frac{1}{k}\left[t+2 l T\left(\alpha_{*}\right)-y_{\alpha_{*}}\left(t+2 l T\left(\alpha_{*}\right)\right)\right]=\frac{1}{k}\left(t-y_{\alpha_{*}}(t)\right), \quad t \in \boldsymbol{R} .
$$

Therefore $u=x$, which is a contradiction.

Remark 4.4. We can also discuss a more general state-dependent functionaldifferential equation

$$
y^{\prime \prime}(t)+a^{2} y\left(t-\frac{k}{a} y(t)\right)=0
$$

where $a, k \in(0, \infty)$. It is easy to check that $x$ is a solution of (1.1) on $\boldsymbol{R}$ if and only if $y(t)=x(a t), t \in \boldsymbol{R}$, is a solution of (4.1) on $\boldsymbol{R}$. Hence our results for periodic solutions of $(1.1)$ can be immediately transformed for periodic solutions of (4.1).

\title{
References
}

[1] Domoshnitsky, A. and Drakhlin, M., Periodic solutions of differential equations with delay depending on solution, Nonlin. Anal., 30 (1997), 2665-2672.

[2] Domoshnitsky, A., Drakhlin, M. and Litsyn, E., On equations with delay depending on solution, Nonlin. Anal., 49 (2002), 689-701.

[ 3 ] Medved, M., On minimal periods of functional-differential equations and difference inclusions, Ann. Polon. Math., 54 (1991), 263-270.

[4] Magal, P. and Arino, O., Existence of periodic solutions for a state dependent delay differential equation, J. Differ. Equations, 165 (2000), 61-95.

[5] Xiang, X. and Ge, W., On the periodic solutions to the differential-iterative equation $x^{\prime}(t)=\omega(t)(a x(t)-b x(x(t))) \quad(a>b>0), \quad$ J. Syst. Sci. Math. Sci., 19 (1999), 457-464. (Chinese)

[6] Yang, W. and Ge, W., Periodic solutions for the differential-iterative equation $x^{\prime \prime}+g(x(x))=p(t)$. J. Beijing Inst. Technol., Chin. Ed., 22 (2002), 537-539. (Chinese)

\author{
nuna adreso: \\ Department of Mathematical Analysis \\ Faculty of Science \\ Palacký University \\ Tomkova 40, 77900 Olomouc \\ Czech Republic \\ E-mail: stanek@inf.upol.cz
}

(Ricevita la 22-an de novembro, 2004)

(Reviziita la 9-an de marto, 2005) 